Check for updates

Cite this: RSC Adv., 2018, 8, 12428

Received 12th February 2018

Accepted 19th March 2018

DOI: $10.1039 / c 8 r a 01343 h$

rsc.li/rsc-advances

\section{Ag-Au bimetallic nanocomposites stabilized with organic-inorganic hybrid microgels: synthesis and their regulated optical and catalytic properties $\uparrow$}

\begin{abstract}
Lei Li, Rui Niu and Ying Zhang (DD *
Herein, we present the synthesis of Ag-Au bimetallic nanocomposites stabilized with organic-inorganic hybrid microgels. The aim is to get both the surface plasmon resonance (SPR) and catalytic performance of the composite material can be changed in response to external stimuli. $\operatorname{Agapoly}(N-$ isopropylacrylamide-co-3-methacryloxypro-pyltrimethoxysilane) (Ag@P(NIPAM-co-MAPTMS)) hybrid microgels were synthesized by seed-emulsion polymerization using Ag nanoparticles (NPs) as the core and NIPAM/MAPTMS as monomers. Ag-Au@P(NIPAM-CO-MAPTMS) bimetallic hybrid microgels were prepared by a galvanic replacement (GR) reaction between Ag NPs and $\mathrm{HAuCl}_{4}$, with the composition and structure of these bimetallic nanocomposites being determined by the amount of added $\mathrm{HAuCl}_{4}$. The highly porous organic-inorganic microgel layer provided confined space for the GR reaction, effectively preventing the aggregation of Ag-Au NPs. The shell layer of P(NIPAM-co-MAPTMS) threedimensional network chains not only enhanced nanocomposite dispersity and stability, but also provided highly porous gel microdomains that could increase the diffusion of the substrate and hence enhanced catalytic activity. Additionally, the SPR and catalytic properties of Ag-Au@P(NIPAM-CO-MAPTMS) are reversibly sensitive to external temperature. With increase of temperature, the maximum absorption peak of bimetallic nanocomposites shifted to longer wavelengths, and the catalytic activity of these composites for the reduction of 4-nitrophenol by $\mathrm{NaBH}_{4}$ remarkably increased. The features above mentioned are related to presence of the thermosensitive PNIPAM chains and the highly porous structure constructed by rigid MAPTMS segments intersected between NIPAM chains.
\end{abstract}

\section{Introduction}

Bimetallic nanoparticles (NPs) have been widely used in catalysis, sensing, and biomedical applications ${ }^{1-3}$ owing to their multi-functionality originating from the synergistic effects of the two components, exhibiting an increased number of active sites due to the enhanced effect of the electromagnetic field on bimetallic interfaces. ${ }^{4-7}$ In addition, the above NPs exhibit enhanced catalytic activity and selectivity owing to their ability to improve electron transfer rates and change original electronic structures. ${ }^{8-10}$ Meanwhile, the regulation of bimetallic NPs morphology/crystal structure can greatly enhance surface plasmon resonance (SPR) properties and hence improve catalytic performance, optical absorption, and surface-enhanced Raman scattering. ${ }^{\mathbf{1 1 - 1 3}}$ Among the multitude of bimetallic NPs, $\mathrm{Ag}-\mathrm{Au}$ ones have attracted increased attention due to the high extinction coefficient and electronegativity of Ag NPs and the

Key Laboratory of Applied Surface and Colloid Chemistry, Ministry of Education, School of Chemistry and Chemical Engineering, Shaanxi Normal University, Xi'an 710062, P. R. China. E-mail: zhangy@snnu.edu.cn

$\dagger$ Electronic supplementary information (ESI) available. See DOI: 10.1039/c8ra01343h high chemical stability and biological activity of Au NPs. ${ }^{\mathbf{1 4 , 1 5}}$ The similarity between the lattice spacings of $\mathrm{Ag}$ and $\mathrm{Au}$ allows the fabrication of $\mathrm{Ag}-\mathrm{Au}$ bimetallic nanocrystals with tunable morphology, ${ }^{16}$ and the optical performance of $\mathrm{Ag}-\mathrm{Au}$ NPs can be regulated by adjusting their structure and the relative contents of $\mathrm{Ag}$ and $\mathrm{Au} .{ }^{\mathbf{1 7}, 18}$

Compared to the co-reduction approach, the galvanic replacement $(\mathrm{GR})$ reaction strategy had received more attention due to offering facile reaction conditions and allowing the fabrication of bimetallic NPs with tunable structures that can be controlled by adjusting the concentration ratio of metal precursor salts. ${ }^{19-21}$ The unique porous structure of $\mathrm{Ag}-\mathrm{Au}$ bimetallic NPs is characterized by an increased number of surface active sites and a large void volume, which not only promotes electron transfer on the nanocatalyst surface, but also accelerates the reaction of the substrate in contact with the catalyst and thus improves catalytic activity. ${ }^{22-24}$

The surface of bimetallic NPs is commonly coated with chemically inert protective layers $\left(\mathrm{SiO}_{2}\right.$, dendrimers, polymers, etc.) to improve dispersity and stability. ${ }^{25-27}$ In particular, environment-sensitive polymer microgels with threedimensional network structures can not only effectively inhibit the aggregation of metal NPs, but also make their 
optical and catalytic properties sensitive to changes of environmental conditions such as temperature, $\mathrm{pH}$, and ionic strength. ${ }^{28-31}$ Among the variety of environment-sensitive polymer microgels, poly( $N$-isopropylacrylamide) (PNIPAM) has received considerable attention due to its low critical solution temperature (LCST) being close to human body temperature. ${ }^{32}$ For example, Liz-Marzán et al. have developed Au-PNIPAM core-shell nanocomposites, showing that the catalytic activity of encapsulated Au NPs is affected by both temperature and shell composition. Importantly, the diffusion of reactants toward the catalytic core can be tuned by the closure/opening of the PNIPAM network in response to temperature changes. ${ }^{33} \mathrm{Lu}$ et al. synthetized yolk-shell $\mathrm{Au}-$ PNIPAM hybrid microgels wherein Au NPs were encapsulated in a hollow PNIPAM shell, with the porosity and hydrophobicity being effectively controlled by temperature changes. Moreover, the above Au NPs exhibited tunable catalytic activity and selectivity in the reduction of 4-nitrophenol (4-NP) and nitrobenzene (NB) in aqueous solution. ${ }^{34} \mathrm{Wu}$ et al. reported a one-pot synthesis of core-shell Au@polyvinylpyrrolidone (PVP) hybrid nanogels, revealing that embedded Au NPs demonstrated both $\mathrm{pH}$-modulated catalytic activity and antiaggregation properties upon recycling. ${ }^{35}$ Zha et al. reported a novel type of three-component composite microgels (AuNR@(AgNPs/PNIPAM)), showing that they exhibit lightcontrollable catalytic activity for the reduction of 4-NP by $\mathrm{NaBH}_{4}$ that can be modulated by varying the power density of visible and near-infrared (NIR) light. ${ }^{36}$ Ahmed and Farooqi have reported Ag NPs were introduced within the copolymerization of NIPAM with acrylamide (AAm), or 2-hydroxyethylmethacrylate (HEMA) and acrylic acid (AAc) hybrid microgels. It was showed that these polymer microgels do not only stabilize Ag NPs in polymer network but also enhance the mass transport of hydrophilic reactant 4-NP from outside to inside the polymer network. ${ }^{37-39}$

Compared to pure PNIPAM network chains, the swelling and shrinkage of $\operatorname{poly}(\mathrm{N}$-isopropylacrylamide-co-3-methacryloxypropyltrimethoxysilane) (P(NIPAM-co-MAPTMS)) organicinorganic hybrid microgels with temperature was partly limited because the thermosensitive PNIPAM chains were separated by rigid MAPTMS segments. ${ }^{\mathbf{4 0 4 1}}$ Furthermore, the rigid MAPTMS segments could enhance the three-dimensional porous inflexibility of P(NIPAM-co-MAPTMS), which is favor for the diffusion and mass transfer. We herein propose a novel route for the fabrication of porous $\mathrm{Ag}-\mathrm{Au}$ bimetallic nanocomposites stabilized by organic-inorganic hybrid microgels with temperature sensitivity, and these structural features are expected to enhance the catalytic reactivity of bimetallic nanoparticles by intensification of the mass transformation. Based on above considerations, Ag@P(NIPAM-co-MAPTMS) hybrid microgels were synthesized by seed-emulsion polymerization using Ag NPs as the core and NIPAM/MAPTMS as monomers. Furthermore, $\mathrm{Ag}-\mathrm{Au}$ bimetallic nanocomposites stabilized with $\mathrm{P}$ (NIPAM-co-MAPTMS) organic-inorganic hybrid microgels were prepared by a GR reaction between Ag NPs and $\mathrm{HAuCl}_{4}$, exhibiting SPR and catalytic properties being sensitive to external temperature. The shell layer of P(NIPAM-co-MAPTMS) three-dimensional network chains not only enhanced nanocomposite dispersity and stability, but also provided highly porous gel microdomains that could increase the diffusion of the substrate and hence enhanced catalytic activity. These expectations were proved through the reduction of 4-NP by $\mathrm{NaBH}_{4}$ catalyzed by Ag-Au@P(NIPAM-co-MAPTMS) bimetallic hybrid microgels.

\section{Experimental}

\section{Materials}

NIPAM (TCI) was purified by recrystallization from $n$-hexane/ benzene $(40 / 60, v / v)$ before use. MAPTMS was supplied by Alfa Aesar. $\operatorname{PVP}\left(M_{\mathrm{w}} \approx 30000\right)$, sodium citrate, glucose, silver nitrate $\left(\mathrm{AgNO} \mathrm{N}_{3}\right), \mathrm{NH}_{3} \cdot \mathrm{H}_{2} \mathrm{O}$ (25 wt\%), sodium dodecyl sulfate (SDS), $N, N^{\prime}$-methylenebisacrylamide (MBA), ammonium persulfate (APS), chloroauric acid $\left(\mathrm{HAuCl}_{4}, 99.3 \%\right)$, absolute ethanol, sodium borohydride $\left(\mathrm{NaBH}_{4}\right), 4-\mathrm{NP}$, and sodium hydroxide $(\mathrm{NaOH})$ were purchased from Sinopharm Chemical Reagent Co. (China) and used as received. Doubly distilled water was used in all experiments.

\section{Synthesis of Ag@P(NIPAM-co-MAPTMS) hybrid microgels}

Ag NPs stabilized by P(NIPAM-co-MAPTMS) organic-inorganic hybrid microgels were prepared in two steps.

In the first step, Ag NPs were prepared using glucose as a reductant and sodium citrate/PVP as stabilizers. ${ }^{42}$ Typically, a solution of $0.151 \mathrm{~g}$ of PVP, $0.048 \mathrm{~g}$ of sodium citrate, and $0.082 \mathrm{~g}$ of glucose in $30 \mathrm{~mL}$ of water was transferred into a $100 \mathrm{~mL}$ three-necked round-bottom flask and stirred at $400 \mathrm{rpm}$ while being purged with $\mathrm{N}_{2}$ to remove oxygen. The deoxygenated solution was heated to $100{ }^{\circ} \mathrm{C}$, and fresh $\left[\mathrm{Ag}\left(\mathrm{NH}_{3}\right)_{2}\right]^{+}$was dropwise added within $30 \mathrm{~min}$ to obtain a green-yellow Ag NP dispersion that was repeatedly centrifuged with doubly distilled water at $9500 \mathrm{rpm}$ for $30 \mathrm{~min}$. Finally, the obtained NP dispersion was diluted with water to $30 \mathrm{~mL}$.

$\mathrm{Ag}$ NPs encapsulated in P(NIPAM-co-MAPTMS) organicinorganic hybrid microgels were synthesized by seeded emulsion polymerization using Ag NPs modified with NIPAM as core seeds. Typically, $10 \mathrm{~mL}$ of Ag NP dispersion was added to a solution of $0.08 \mathrm{~g}$ of NIPAM and $0.018 \mathrm{~g}$ of SDS in $50 \mathrm{~mL}$ of water, and the mixture was stirred for $24 \mathrm{~h}$, being subsequently heated to $70{ }^{\circ} \mathrm{C}$ under a flow of $\mathrm{N}_{2}$ while stirring at $400 \mathrm{rpm}$ in a $150 \mathrm{~mL}$ three-necked round-bottom flask equipped with a condenser. In the next step, an aqueous solution of an appropriate amount of NIPAM and $0.003 \mathrm{~g}$ of MBA cross-linker was added to the reaction flask. After $1 \mathrm{~h}, 28 \mu \mathrm{L}$ of aqueous APS $(0.95 \mathrm{M})$ and $0.005 \mathrm{~g}$ of MAPTMS were injected into the reaction system, and polymerization was performed for $4 \mathrm{~h}$. The produced yellow Ag@P(NIPAM-co-MAPTMS) hybrid microgels were repeatedly centrifuged at $9500 \mathrm{rpm}$, sequentially washed with alternately water and absolute ethanol, and dispersed in $50 \mathrm{~mL}$ of water before use. To determine the effect of NIPAM loading on the dispersity of the Ag@P(NIPAM-co-MAPTMS) hybrid microgel shell layer, P(NIPAM-co-MAPTMS) organicinorganic hybrid microgels were fabricated using different 
masses of NIPAM $(0.02,0.04,0.06$, and $0.08 \mathrm{~g})$ while keeping the amounts of MAPTMS, MBA, APS, and SDS constant.

\section{Synthesis of Ag-Au@P(NIPAM-co-MAPTMS) bimetallic hybrid microgels}

Core-shell Ag-Au@P(NIPAM-co-MAPTMS) bimetallic hybrid microgels were controllably synthesized by a GR reaction between $\mathrm{Ag}$ NPs and $\mathrm{HAuCl}_{4}$ using the former as a sacrificial template (Fig. 1). Typically, 50 mL of Ag@P(NIPAM-co-MAPTMS) aqueous dispersion was added into a $150 \mathrm{~mL}$ three-necked round-bottom flask upon $30 \mathrm{~min}$ stirring $(400 \mathrm{rpm})$ at $25{ }^{\circ} \mathrm{C}$. Subsequently, a certain volume of aqueous $\mathrm{HAuCl}_{4}(0.002 \mathrm{M})$ was added into the flask using a constant-pressure funnel. After $6 \mathrm{~h}, 1.6 \mathrm{~mL}$ of aqueous ammonia was added into the flask, and the reaction was continued for $30 \mathrm{~min}$. The obtained $\mathrm{Ag}$ $\mathrm{Au} @ \mathrm{P}(\mathrm{NIPAM}-\mathrm{co}$-MAPTMS) bimetallic hybrid microgels were centrifuged (9000 rpm), sequentially washed alternately with water and absolute ethanol for several times, and freeze-dried (FD-1A-80, Beijing Boyikang Experimental Equipment Company).

Ag-Au@P(NIPAM-co-MAPTMS) bimetallic hybrid microgels with different contents of $\mathrm{Ag}$ and $\mathrm{Au}$ were controllably prepared by changing the volume of $\mathrm{HAuCl}_{4}$ solution $(1.0,3.0,6.0$, and $9.0 \mathrm{~mL}$ ), with the utilized nomenclature presented in Table 1.

\section{Characterization}

The morphology and size dispersion of hybrid microgels were probed by transmission electron microscopy (TEM; JEM-2100, JEOL, Japan) at an accelerating voltage of $200 \mathrm{kV}$. Elemental mapping and high-angle annular dark field scanning TEM (HAADF-STEM) imaging were conducted using field emission transmission electron microscopy (FE-TEM) coupled with energy-dispersive X-ray spectroscopy (EDX) (Tecnai G2 F20, FEI, USA). UV-Vis spectra were recorded on a U-3900 UV-Vis spectrophotometer equipped with a temperature controller (Hitachi, Japan). Infrared spectra were recorded on an Avatar 360 Fourier transform infrared (FT-IR) spectrometer (Nicolet, USA) using the $\mathrm{KBr}$ pellet technique. Thermal stabilities were determined using a Q1000DSC+LNCS+FACS Q600SDT thermogravimetric analyzer (TA, USA) at a heating rate of $10{ }^{\circ} \mathrm{C} \mathrm{min}^{-1}$ in an atmosphere of $\mathrm{N}_{2}$. Swelling behavior was characterized by dynamic light scattering measurements (Nano-ZS90,

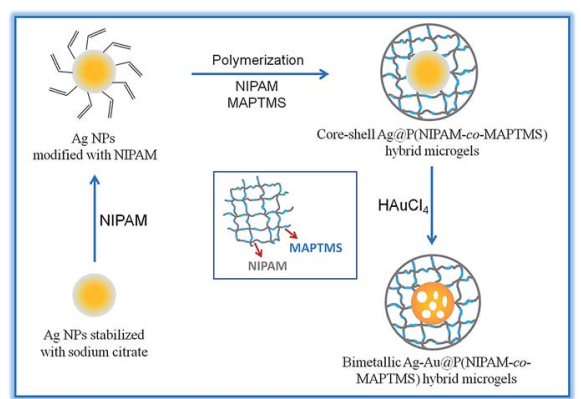

Fig. 1 Schematic illustration for synthesis of core-shell AgAu@P(NIPAM-CO-MAPTMS) bimetallic hybrid microgels.
Table 1 Naming of Ag-Au@P(NIPAM-Co-MAPTMS) bimetallic hybrid microgels prepared using different amounts of $\mathrm{HAuCl}_{4}$

Samples Volume of $\mathrm{HAuCl}_{4}$

Ag@P(NIPAM-co-MAPTMS)

$0 \mathrm{~mL}$

Ag-Au@P(NIPAM-co-MAPTMS)-1

$\mathrm{Ag}-\mathrm{Au} @ \mathrm{P}(\mathrm{NIPAM}-\mathrm{co}$-MAPTMS)-2

$\mathrm{Ag}-\mathrm{Au} @ \mathrm{P}(\mathrm{NIPAM}-c o-\mathrm{MAPTMS})-3$

Ag-Au@P(NIPAM-co-MAPTMS)-4

$1 \mathrm{~mL}$

$3 \mathrm{~mL}$

$6 \mathrm{~mL}$

$9 \mathrm{~mL}$

Malvern, UK) in the temperature range of $25-45{ }^{\circ} \mathrm{C}$. Crystal structures were determined by X-ray diffraction (XRD) analysis (D/MAX-3C, Rigaku, Japan) performed using $\mathrm{Cu} \mathrm{K}_{\alpha}$ radiation at $35 \mathrm{kV}$ and $40 \mathrm{~mA}$. Surface compositions were determined by Xray photoelectron spectroscopy (XPS; AXIS ULTRA, Kratos Analytical Ltd., Japan) using monochromatic $\mathrm{Al} \mathrm{K}_{\alpha}$ radiation.

\section{Catalytic reduction of 4-NP}

The catalytic activity of hybrid microgels was systematically evaluated for the reduction of 4-NP by excess $\mathrm{NaBH}_{4}$ as a model reaction, and the reaction progress was monitored in situ by measuring changes of 4-NP absorbance at $400 \mathrm{~nm}$. Typically, a $0.003 \mathrm{~g}$ catalyst sample was dissolved in $20 \mathrm{~mL}$ of water. Separately, $100 \mu \mathrm{L}$ of aqueous 4 -NP $\left(2.0 \times 10^{-3} \mathrm{M}\right)$ and $3.0 \mathrm{~mL}$ of aqueous $\mathrm{NaBH}_{4}(0.10 \mathrm{M})$ were added into a standard quartz cuvette (solution $\mathrm{pH}$ was adjusted to 10 by $\mathrm{NaOH}$ ), and $80 \mu \mathrm{L}$ of the above catalyst dispersion was injected. The absorption spectra of the obtained mixture were acquired in situ in the range of 250-500 $\mathrm{nm}$ at $1 \mathrm{~min}$ intervals for different reaction temperatures, and apparent reaction rate constants $\left(k_{\text {app }}\right)$ were obtained from temporal changes of the absorption peak intensity at $400 \mathrm{~nm}$ by plotting $\ln \left(A_{t} / A_{0}\right) v s$. reduction time ( $A_{t}$ and $A_{0}$ are absorbances at time $t$ and 0 , respectively). ${ }^{43}$

\section{Results and discussion}

\section{Morphology and crystal structure of Ag NPs}

TEM imaging showed that the prepared bright yellow Ag NPs exhibited a quasi-spherical shape (Fig. 2a and b) with an average diameter of $\sim 42 \mathrm{~nm}$ (Fig. 2c) and a maximum absorption wavelength of $423 \mathrm{~nm}$ (Fig. 2d), which was attributed to their SPR. ${ }^{44}$ Furthermore, the narrow half peak width of the UV-Vis absorption peak confirmed that Ag NPs exhibited strong SPR. High-resolution TEM imaging allowed the lattice spacing $d$ to be determined as $\sim 0.235 \mathrm{~nm}$ (Fig. 2e), which was assigned to the (111) crystal planes of face-centered cubic Ag. ${ }^{45}$ Fig. $2 \mathrm{f}$ shows a typical XRD pattern of Ag NPs, with diffraction peaks at $38.2^{\circ}$, $44.4^{\circ}, 64.5^{\circ}$, and $77.6^{\circ}$ corresponding to the (111), (200), (220), and (311) planes of face-centered cubic Ag, ${ }^{46}$ respectively. The dominance of the (111) peak suggested that the obtained $\mathrm{Ag}$ NPs mainly featured (111) facets. ${ }^{45}$

\section{Morphology of Ag@P(NIPAM-co-MAPTMS) hybrid microgels}

Ag NPs stabilized with P(NIPAM-co-MAPTMS) organic-inorganic hybrid microgels were synthesized by seed-emulsion 

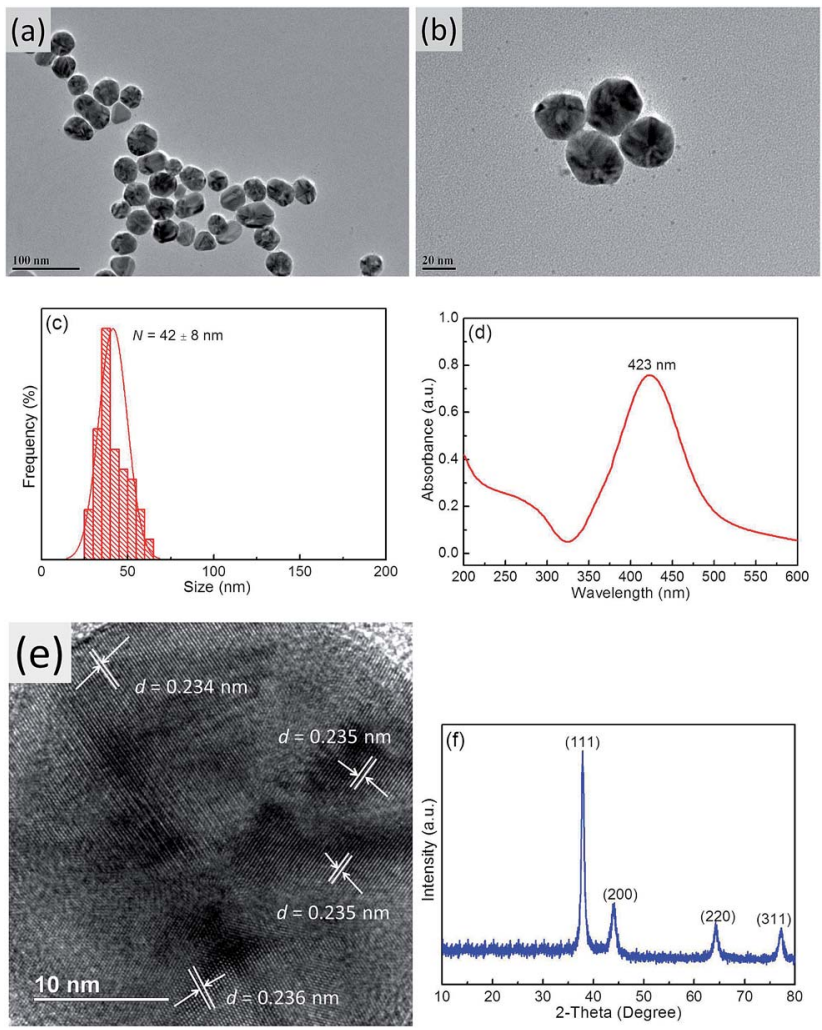

Fig. 2 TEM images ( $a$ and b), size distribution (c), UV-Vis spectrum (d), HR-TEM image (e), and XRD pattern (f) of Ag NPs.

polymerization using NIPAM-modified Ag NPs as core seeds, with the amount of the NIPAM monomer directly affecting the dispersity and uniformity of the P(NIPAM-co-MAPTMS) coating layer. At a NIPAM loading of $0.02 \mathrm{~g}$, the obtained surface layer was uneven, and the Ag NPs were prone to aggregation (ESI, Fig. S1a and $\mathrm{b} \dagger$ ). Conversely, a much better coating layer was observed at a NIPAM loading of $0.04 \mathrm{~g}$ (Fig. $3 \mathrm{a}$ and $\mathrm{b}$ ), and the thus obtained Ag@P(NIPAM-co-MAPTMS) hybrid microgels exhibited good dispersity and a well-pronounced core-shell structure. The average hydrodynamic diameter of the above microgels was determined as $\sim 243 \mathrm{~nm}$ by dynamic light scattering measurements (Fig. 3c). However, at increased NIPAM loadings $(0.06$ and $0.008 \mathrm{~g}$ ), large numbers of individual $\mathrm{P}$ (NIPAM- $c o$-MAPTMS) particles were formed due to the monomers being present in excess to Ag NPs (ESI, Fig. S1c-f

Compared to that of Ag NPs (423 nm), the absorption peak maximum of Ag@P(NIPAM-co-MAPTMS) hybrid microgels was red-shifted to $446 \mathrm{~nm}$ (Fig. 4) due to the cross-linked structure of P(NIPAM-co-MAPTMS) shell layers altering the refractive index of coated Ag NPs and hence enhancing their Rayleigh scattering and inducing a red shift of the SPR absorption peak. ${ }^{47}$

The presence of PNIPAM and MAPTMS in organic-inorganic hybrid microgels was confirmed by FT-IR spectroscopy (ESI, Fig. S2†). Peaks at 2877,2935 , and $2935 \mathrm{~cm}^{-1}$ were assigned to $\mathrm{C}-\mathrm{H}$ stretching vibrations and $-\mathrm{CH}_{2}$ and $-\mathrm{CH}_{3}$ groups of PNI$\mathrm{PAM},{ }^{48}$ while those at 1649 and $1547 \mathrm{~cm}^{-1}$ were assigned to the $\mathrm{C}=\mathrm{O}$ stretch of PNIPAM amide (I) and (II) groups. The peak at
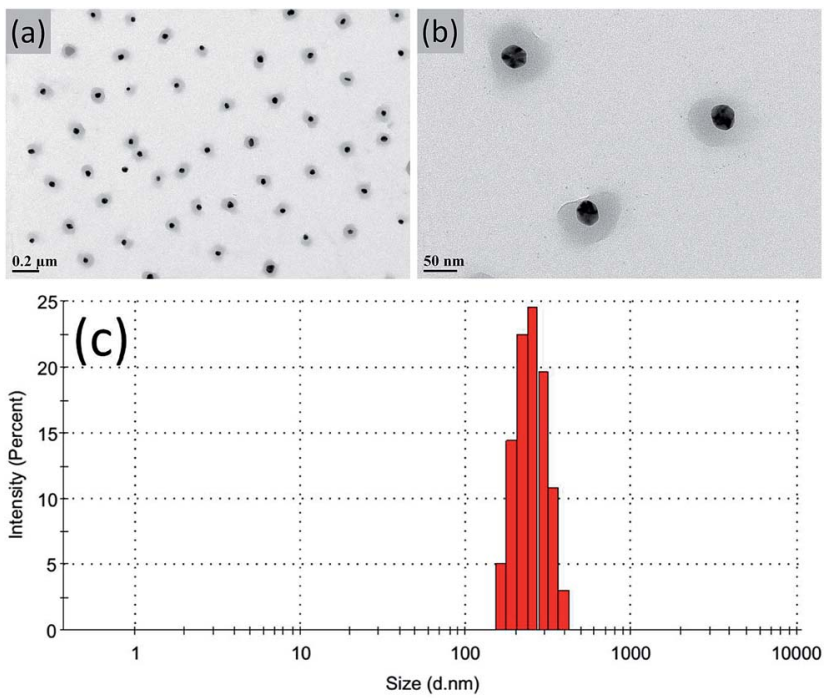

Fig. 3 TEM images ( $a$ and $b$ ) and hydrodynamic diameter distribution (c) of Ag@P(NIPAM-co-MAPTMS) hybrid microgels prepared at a NIPAM loading of $0.04 \mathrm{~g}$.

$1387 \mathrm{~cm}^{-1}$ was attributed to the deformation of PNIPAM $-\mathrm{C}\left(\mathrm{CH}_{3}\right)_{2}$ methyls, and that at $1126 \mathrm{~cm}^{-1}$ was assigned to the $-\mathrm{Si}-\mathrm{O}-\mathrm{Si}$ - stretching vibration of MAPTMS. ${ }^{49}$

\section{Morphology and structure of Ag-Au@P(NIPAM-co-MAPTMS) bimetallic hybrid microgels}

Ag NPs covered with P(NIPAM-co-MAPTMS) organic-inorganic hybrid microgels were used as sacrificial templates to synthesize $\mathrm{Ag}-\mathrm{Au}$ bimetallic nanocomposites via a GR reaction between Ag NPs and $\mathrm{HAuCl}_{4}$. Herein, we chose ammonia for removing $\mathrm{AgCl}$ produced as a by-product during the formation of $\mathrm{Au}^{50}{ }^{50} \mathrm{To}$ explore the effect of $\mathrm{HAuCl}_{4}$ dosage on the structure of $\mathrm{Ag}-\mathrm{Au}$ bimetallic nanocomposites, Ag-Au@P(NIPAM-coMAPTMS) hybrid microgels were prepared using different volumes of $0.002 \mathrm{M}$ aqueous $\mathrm{HAuCl}_{4}(1.0,3.0,6.0$, and $9.0 \mathrm{~mL}$ ), with the corresponding samples denoted as Ag-Au@P(NIPAM$c o$-MAPTMS)-1, Ag-Au@P(NIPAM-co-MAPTMS)-2, Ag-Au@P(NIPAM-co-MAPTMS)-3, and Ag-Au@P(NIPAM-co-MAPTMS)-4, respectively (Table 1).

The contents of $\mathrm{Ag}$ and $\mathrm{Au}$ and the structure of $\mathrm{Ag}-\mathrm{Au}$ bimetallic nanocomposites were significantly affected by the

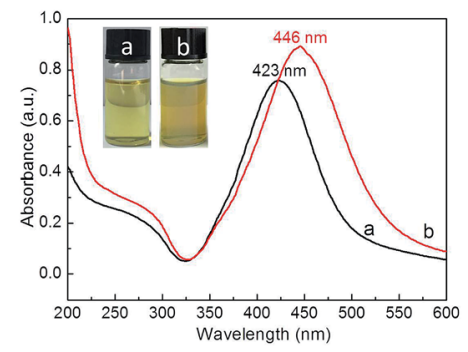

Fig. 4 UV-Vis spectra and photographs of Ag NPs (a) and Ag@P(NIPAM-CO-MAPTMS) hybrid microgels (b). 
dosage of $\mathrm{HAuCl}_{4}$, e.g., the morphology of the above nanocomposites changed from porous to hollow with increasing $\mathrm{HAuCl}_{4}$ solution volume. Thus, when $1.0 \mathrm{~mL}$ of $\mathrm{HAuCl}_{4}$ was used, porous structures were observed (Ag-Au@P(NIPAM-coMAPTMS)-1; Fig. 5a and b), whereas sharp edges appeared when the volume equaled $3.0 \mathrm{~mL}$ (Ag-Au@P(NIPAM-co-MAPTMS)-2; Fig. $5 \mathrm{c}$ and d). As the volume of $\mathrm{HAuCl}_{4}$ increased to $6.0 \mathrm{~mL}$, the holes in the porous structure of Ag-Au@P(NIPAM-coMAPTMS)-3 bimetallic hybrid microgels became larger (Fig. 5e and f), and a well-pronounced hollow structure was finally obtained for Ag-Au@P(NIPAM-co-MAPTMS)-4 (Fig. 5g and h).

To determine the structures of $\mathrm{Ag}$ and $\mathrm{Au}$ in $\mathrm{Ag}-\mathrm{Au} @ \mathrm{P}(\mathrm{NI}-$ PAM-co-MAPTMS)-2, the above nanocomposite was probed by elemental mapping (HAADF-STEM), which showed that Ag and $\mathrm{Au}$ elements were uniformly distributed in the core region of Ag-Au@P(NIPAM-co-MAPTMS)-2 bimetallic hybrid microgels (ESI, Fig. $\mathrm{S} 3 \dagger$ ).

As mentioned above, the structure of $\mathrm{Ag}-\mathrm{Au}$ bimetallic nanocomposites embedded in P(NIPAM-co-MAPTMS) hybrid microgels was regulated by changing the amount of added $\mathrm{HAuCl}_{4}$, which was rationalized as follows. During the GR reaction between $\mathrm{Ag}$ NPs and $\mathrm{HAuCl}_{4}, \mathrm{AuCl}_{4}{ }^{-}$ions acted as electron donors and were adsorbed on the surface of Ag NPs. The addition of a small amount of $\mathrm{Au}$ initially led to the formation of pits on the $\mathrm{Ag}$ surface that subsequently evolved
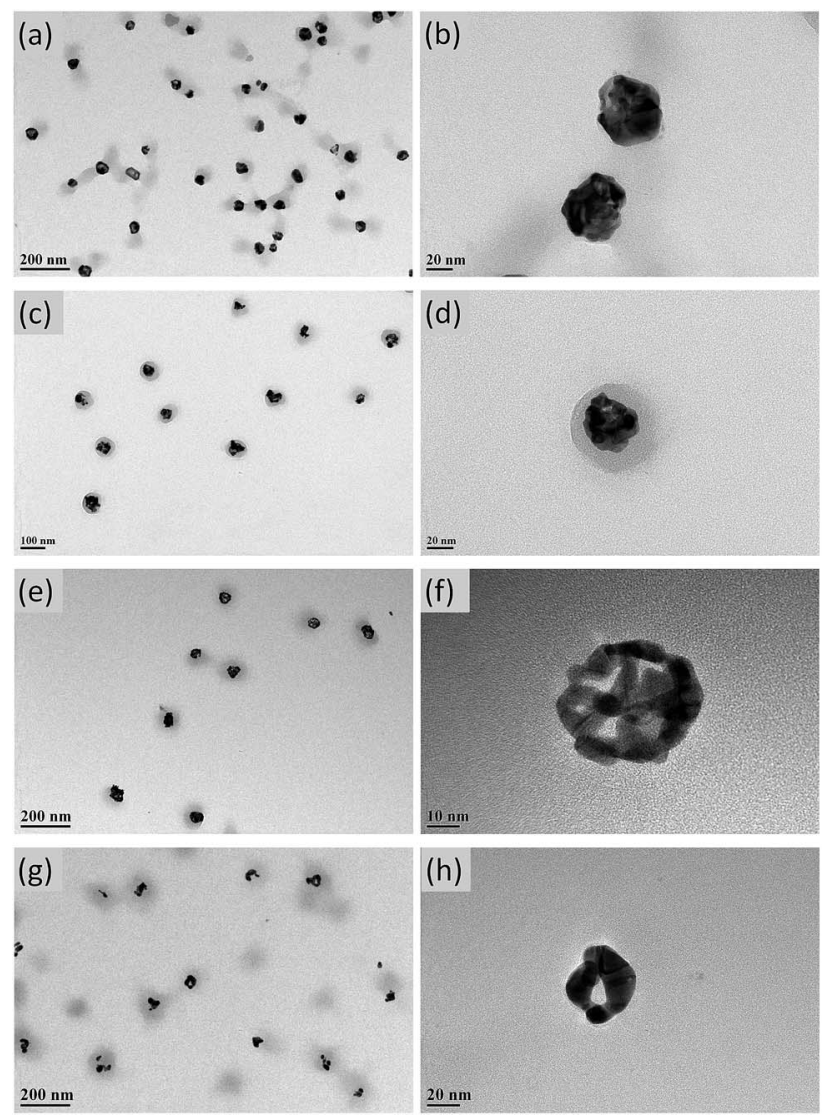

Fig. 5 TEM images of Ag-Au@P(NIPAM-co-MAPTMS) bimetallic hybrid microgels obtained at different volumes of $\mathrm{HAuCl}_{4}$ solution. (a and b) $1.0 \mathrm{~mL}$, (c and d) $3.0 \mathrm{~mL}$, (e and f) $6.0 \mathrm{~mL}$, (g and h) $9.0 \mathrm{~mL}$.

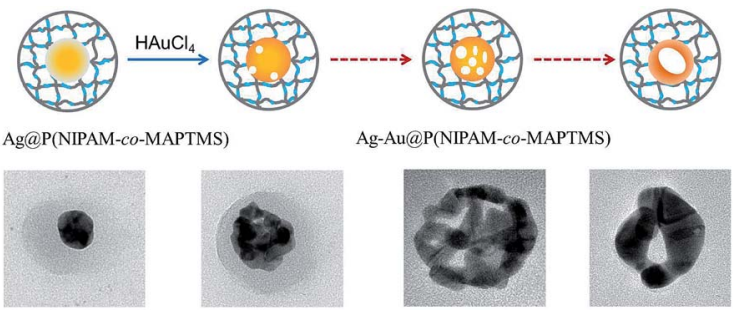

Fig. 6 The schematic illustration of the possible mechanism for AgAu bimetallic nanocomposites with pore structure by GR reaction.

into voids of $\mathrm{Ag}-\mathrm{Au}$ bimetallic nanocomposites due to the dissolution of $\mathrm{Ag}$ in the particle interior. Thus, at increased amounts of $\mathrm{AuCl}_{4}{ }^{-}$ions, $\mathrm{Au}-\mathrm{Ag}$ bimetallic nanocomposites with hollow interiors and porous walls formed as a result of $\mathrm{Ag}$ dealloying from $\mathrm{Au}-\mathrm{Ag}$ alloyed walls. The formation of these hollow structures was attributed to the difference of $\mathrm{Ag}$ and $\mathrm{Au}$ diffusion rates, which is known as the Kirkendall effect, ${ }^{21}$ with the overall mechanism schematically illustrated in Fig. 6.

The crystal structures of Ag-Au@P(NIPAM-co-MAPTMS) bimetallic hybrid microgels with different $\mathrm{Ag}$ and $\mathrm{Au}$ contents were determined by HR-TEM. Based on the crystal lattice spacings in different regions of Ag-Au@P(NIPAM-co-MAPTMS) (ESI, Fig. S4 $\dagger$ ), their two lattice spacing were determined as 0.202 and $0.233 \mathrm{~nm}$, being similar to those between $\mathrm{Ag}$ and $\mathrm{Au}$ (200) and (111). ${ }^{51}$ In addition, the Fast Fourier Transform (FFT) maps of different Ag-Au@P(NIPAM-co-MAPTMS) bimetallic hybrid microgels showed polycrystalline rings and thus indicated that the formed nanocomposites exhibited a polycrystalline structure. ${ }^{9}$

The crystal structures of composite materials were investigated by XRD (Fig. 7). For Ag@P(NIPAM-co-MAPTMS), the broad peak at $\sim 20^{\circ}$ was assigned to amorphous P(NIPAM-coMAPTMS) shell layers. Compared to those of Ag@P(NIPAM-coMAPTMS) (Fig. 7a), the peaks of Ag-Au@P(NIPAM-co-MAPTMS) at $38.1^{\circ}, 44.3^{\circ}, 64.4^{\circ}$, and $77.4^{\circ}$ corresponding to Au or Ag NPs (face-centered cubic, JCPDF 04-783 and 04-784) and the diffraction peaks of $\mathrm{Au}$ and $\mathrm{Ag}$ were obviously weakened (Fig. 7b), which indicated the occurrence of Ag-Au alloying. ${ }^{52}$

The chemical compositions of the prepared microgels were further probed by XPS. Both Ag@P(NIPAM-co-MAPTMS) (Fig. 8A-a) and Ag-Au@P(NIPAM-co-MAPTMS)-3 showed typical signals of $\mathrm{C}, \mathrm{N}, \mathrm{O}, \mathrm{Ag}$, and $\mathrm{Si}$, with an additional signal

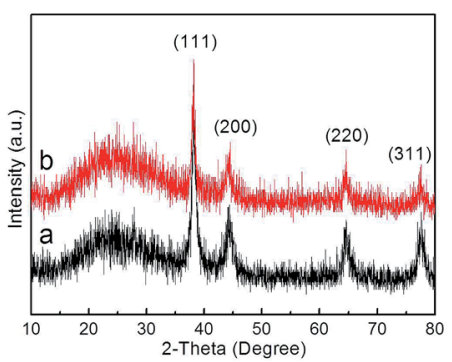

Fig. 7 XRD patterns of the hybrid microgels. (a) AgaP(NIPAM-coMAPTMS), (b) Ag-Au@P(NIPAM-Co-MAPTMS)-3. 

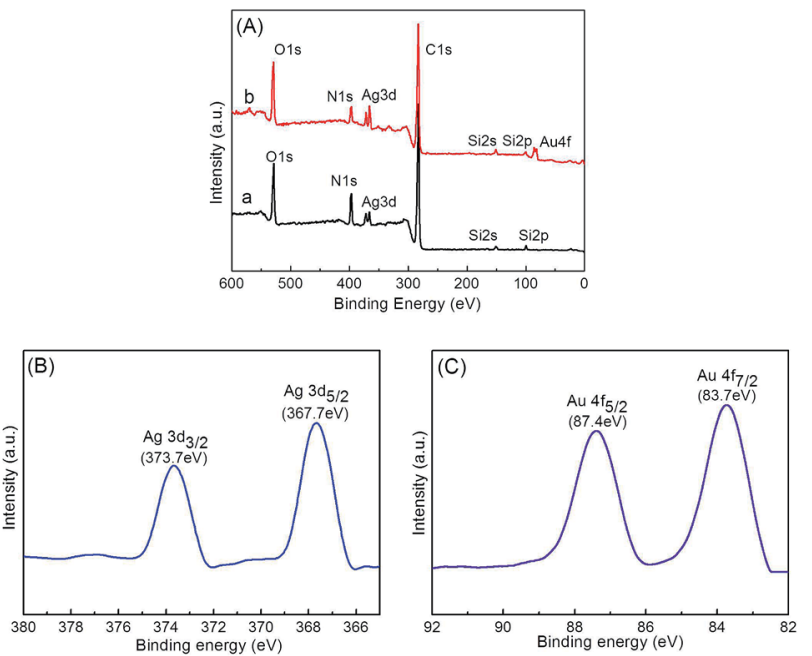

Fig. 8 Survey XPS spectra of hybrid microgels (A) and $\mathrm{Ag} 3 \mathrm{~d}(\mathrm{~B})$ and $\mathrm{Au}$ $4 f(C)$ spectra of Ag-Au(aP(NIPAM-Co-MAPTMS)-3. (a) Ag@P(NIPAMco-MAPTMS), (b) Ag-Au(aP(NIPAM-co-MAPTMS)-3.

of $\mathrm{Au}$ observed for the latter microgel (Fig. 8A-b). The two peaks at 373.5 and $367.5 \mathrm{eV}$ in the high-resolution XPS spectrum of Ag-Au@P(NIPAM-co-MAPTMS)-3 were ascribed to Ag $3 \mathrm{~d}_{3 / 2}$ and $\mathrm{Ag} 3 \mathrm{~d}_{5 / 2}$ transitions, respectively (Fig. 8B). The splitting of the $\mathrm{Ag} 3 \mathrm{~d}$ doublet was estimated as $6.0 \mathrm{eV}$, confirming the metallic nature of $\mathrm{Ag} .{ }^{53}$ The two peaks at 87.4 and $83.7 \mathrm{eV}$ were assigned to $\mathrm{Au} 4 \mathrm{f}_{5 / 2}$ and $\mathrm{Au} 4 \mathrm{f}_{7 / 2}$ transitions, respectively, with the splitting of the $\mathrm{Au} 4 \mathrm{f}$ doublet $(3.7 \mathrm{eV}$; Fig. 8C) also confirming the metallic nature of $\mathrm{Au}^{54}$

Comparison of $\mathrm{Ag} 3 \mathrm{~d}$ and $\mathrm{Au} 4 \mathrm{f}$ spectra of $\mathrm{Ag}-\mathrm{Au} @ \mathrm{P}(\mathrm{NI}-$ PAM-co-MAPTMS) bimetallic hybrid microgels showed that the binding energies of the corresponding peaks decreased with increasing Au content (Fig. 9). Thus, when the volume of $\mathrm{HAuCl}_{4}$ increased from 3 to $9 \mathrm{~mL}$, the binding energies of $\mathrm{Ag}$ $3 \mathrm{~d}_{5 / 2}$ and $\mathrm{Ag} 3 \mathrm{~d}_{3 / 2}$ peaks decreased from 368 to $367.5 \mathrm{eV}$ and from 374 to $373.5 \mathrm{eV}$, respectively (Fig. 9A), with the binding energies of $A u 4 f_{7 / 2}$ and $A u 4 f_{5 / 2}$ peaks decreasing from 84 to $83.5 \mathrm{eV}$ and from 87.7 to $87.2 \mathrm{eV}$, respectively (Fig. 9B). XPS analyses of Ag-Au@P(NIPAM-co-MAPTMS) bimetallic hybrid microgels with different $\mathrm{Ag}-\mathrm{Au}$ contents showed that with increasing $\mathrm{HAuCl}_{4}$ volume, the $\mathrm{Au}$ content of the $\mathrm{Ag}-\mathrm{Au}$ alloy increased, while that of $\mathrm{Ag}^{0}$ decreased and that of $\mathrm{Ag}^{+}$ increased. ${ }^{55}$ The observed binding energy changes of $\mathrm{Ag}$ and
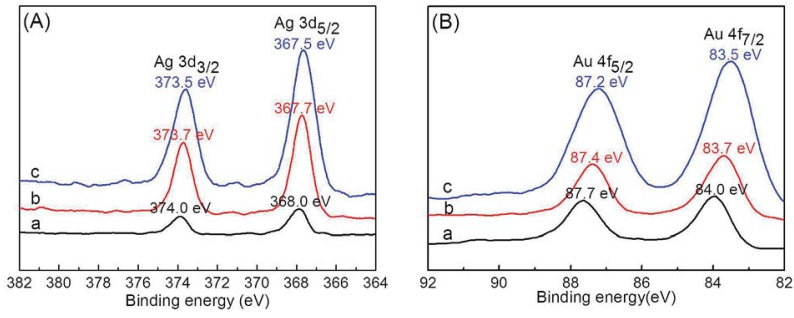

Fig. $9 \mathrm{Ag} 3 \mathrm{~d}(\mathrm{~A})$ and $\mathrm{Au} 4 \mathrm{f}(\mathrm{B})$ XPS spectra of Ag-Au@P(NIPAM-COMAPTMS) bimetallic hybrid microgels prepared using different volumes of $\mathrm{HAuCl}_{4}$. (a) $3.0 \mathrm{~mL}$, (b) $6.0 \mathrm{~mL}$, (c) $9.0 \mathrm{~mL}$.

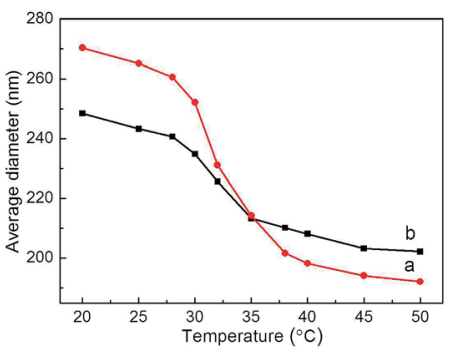

Fig. 10 Variations of microgel hydrodynamic diameter with temperature. (a) Ag@PNIPAM, (b) Ag@P(NIPAM-CO-MAPTMS).

$\mathrm{Au}$ peaks in the spectra of Ag-Au@P(NIPAM-co-MAPTMS) bimetallic hybrid microgels were in accordance with the mechanism of the redox reaction between $\mathrm{Ag}$ NPs and $\mathrm{HAuCl}_{4}$ (ESI, Table S1†).

\section{Thermosensitivity of hybrid microgels}

Hydrodynamic diameter variations of Ag@PNIPAM and Ag@P(NIPAM-co-MAPTMS) were measured by dynamic light scattering at $20-50{ }^{\circ} \mathrm{C}$. Data were acquired after $5 \mathrm{~min}$ sample dispersion stabilization at each temperature, with all measurements repeated five-fold and the final results reported as averages. The swelling degree of temperature-induced of Ag@P(NIPAM-co-MAPTMS) hybrid microgels was less than that of Ag@PNIPAM microgels (Fig. 10). Thus, Ag@PNIPAM microgels featured a hydrodynamic diameter of $270.3 \mathrm{~nm}$ at $20^{\circ} \mathrm{C}$, with the significant decrease to $231.1 \mathrm{~nm}$ observed at $32{ }^{\circ} \mathrm{C}$ (Fig. 10a) rationalized by the fact that the latter temperature corresponded to the LCST of PNIPAM, above which the PNIPAM chains exist in a shrinked state. ${ }^{56}$

The average hydrodynamic diameter of Ag@P(NIPAM-coMAPTMS) equaled $\sim 248.5 \mathrm{~nm}$ at $20^{\circ} \mathrm{C}$, decreasing to $225.7 \mathrm{~nm}$ at $32{ }^{\circ} \mathrm{C}$ (Fig. 10b). Thus, the swelling and shrinkage degrees of the above microgel were obviously reduced by the introduction of rigid MAPTMS segments into polymer chains, which inhibited the swelling and shrinkage of the shell layer ${ }^{40}$ The thermosensitivity of Ag@P(NIPAM-co-MAPTMS) hybrid microgels also decreased after MAPTMS introduction, confirming that the shell layer comprised organic-inorganic network chains.

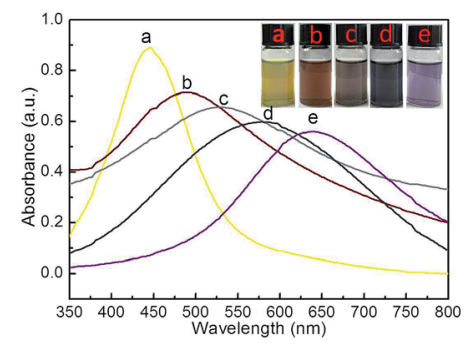

Fig. 11 UV-Vis spectra and photographs of Ag-Au@P(NIPAM-CoMAPTMS) hybrid microgels obtained using different volumes of $\mathrm{HAuCl}_{4}$. (a) $0 \mathrm{~mL}$, (b) $1 \mathrm{~mL}$, (c) $3 \mathrm{~mL}$, (d) $6 \mathrm{~mL}$, (e) $9 \mathrm{~mL}$. 


\section{SPR properties of hybrid microgels}

The UV-Vis spectra of Ag-Au@P(NIPAM-co-MAPTMS) bimetallic hybrid microgels obtained at different dosages of $\mathrm{HAuCl}_{4}$ revealed that the absorption peak maximum at $446 \mathrm{~nm}$ (Fig. 11a) was broadened and shifted to longer wavelengths with increasing $\mathrm{HAuCl}_{4}$ loading $(488,531,578$, and $638 \mathrm{~nm}$ at $1,3,6$, and $9 \mathrm{~mL}$, respectively; Fig. 11b-e), with the concomitant dispersion color change from bright yellow to light purple reflecting the formation of $\mathrm{Ag}-\mathrm{Au}$ alloy nanocomposites at the atomic level. ${ }^{57}$

To study the effect of solvent on the SPR of Ag@P(NIPAM-coMAPTMS) and Ag-Au@P(NIPAM-co-MAPTMS)-3, the UV-Vis absorption spectra of these composites were recorded in water, acetone, tetrahydrofuran, and carbon disulfide (Fig. 12), with the former composite showing absorption maxima at 446, 454, 460, and $467 \mathrm{~nm}$, respectively (Fig. 12a). Similarly, the absorption maxima of Ag-Au@P(NIPAM-co-MAPTMS)-3 progressively shifted to longer wavelengths (Fig. 12b) with increasing solvent refractive index $\left(\mathrm{H}_{2} \mathrm{O}\right.$ 1.333, acetone 1.358, tetrahydrofuran 1.405 , carbon disulfide 1.627$).{ }^{58}$ In addition, the shrinkage of P(NIPAM-co-MAPTMS) in different solvents also changed the refractive index of the micro-environment surrounding $\mathrm{Ag}$ or $\mathrm{Ag}-\mathrm{Au}$ NPs and hence shifted the position of the absorption peak.

The Ag-Au nanocomposites exhibited the environmental regulated LSPR that endowed from the thermosensitivity of P(NIPAM-co-MAPTMS)-based organic-inorganic hybrid microgels, as the temperature was raised from 20 to $50{ }^{\circ} \mathrm{C}$ (with complete microgel collapse observed at the latter temperature), the LSPR peak positions of Ag@P(NIPAM-co-MAPTMS) and AgAu@P(NIPAM-co-MAPTMS)-3 shifted from 446 to $452 \mathrm{~nm}$ and from 578 to $585 \mathrm{~nm}$, respectively (Fig. 13a and b), which was ascribed to the collapse of P(NIPAM-co-MAPTMS) polymer shells resulting in enhanced Rayleigh scattering due to increasing the refractive index contrast with the solvent. The above red shifts of $\mathrm{Ag}$ and $\mathrm{Ag}-\mathrm{Au}$ NPs peaks with increasing temperature were attributed only to the local refractive index increase resulting from water expulsion during heating-induced volume phase transitions. ${ }^{28,47}$ Therefore, it was concluded that the optical properties of $\mathrm{Ag}-\mathrm{Au}$ bimetallic nanocomposites stabilized by P(NIPAM-co-MAPTMS) organic-inorganic hybrid network structures can be regulated by solvent and temperature changes. Moreover, the variation of the SPR band position further confirmed the successful encapsulation of $\mathrm{Ag}$ and $\mathrm{Ag}-\mathrm{Au}$ NPs by the polymer microgel shell.
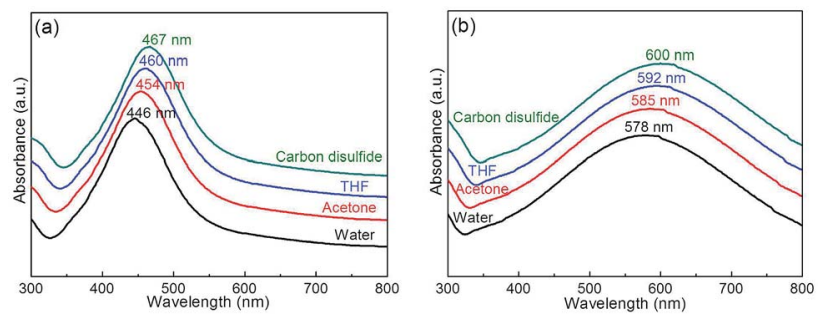

Fig. 12 UV-Vis spectra of hybrid microgels in different solvents. (a) Ag@P(NIPAM-Co-MAPTMS), (b) Ag-Au@P(NIPAM-Co-MAPTMS)-3.
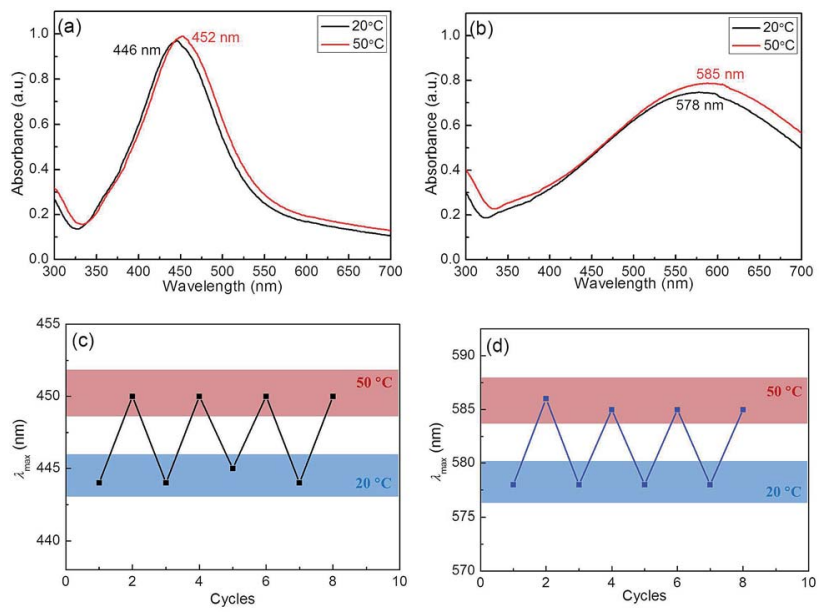

Fig. 13 UV-Vis spectra of hybrid microgels recorded at different temperatures ( $a$ and $b$ ) and extinction peak wavelength change as the hybrid microgels solution was heated up to $50{ }^{\circ} \mathrm{C}$ followed by cooling to $20{ }^{\circ} \mathrm{C}$ for multiple cycles (c and d). (a and c) Ag@P(NIPAM-COMAPTMS), (b and d) Ag-Au@P(NIPAM-CO-MAPTMS)-3.

Fig. 13c shows the values of surface plasmon resonance wavelength of the Ag@P(NIPAM-co-MAPTMS) hybrid microgels as the temperature increases from $20{ }^{\circ} \mathrm{C}$ to $50{ }^{\circ} \mathrm{C}$, followed by cooling down to $20{ }^{\circ} \mathrm{C}$, and then increases again. Initially, at $20{ }^{\circ} \mathrm{C}$, a single peak at $445 \mathrm{~nm}$ of the extinction spectrum shifts to $451 \mathrm{~nm}$ upon heating up to $50^{\circ} \mathrm{C}$. After cooling back to $20^{\circ} \mathrm{C}$, the peak at $451 \mathrm{~nm}$ returns to the previous $445 \mathrm{~nm}$. This change is related to the fact that the hybrid microgel at $20{ }^{\circ} \mathrm{C}$ is in a swelling state, while that at $50{ }^{\circ} \mathrm{C}$ is in a shrinking state. Generally, the shrinkage makes refractive index increase, resulting in absorption spectrum red shift. It is this reason that the SPR peak of Ag NPs shows red shift as the temperature increase from $20{ }^{\circ} \mathrm{C}$ to $50{ }^{\circ} \mathrm{C}$. Similarly, Ag-Au@P(NIPAM-coMAPTMS)-3 hybrid microgels also showed SPR variation with temperature change (Fig. 13d). In fact, the shrinkage of the microgel could lead to a decrease of distance between Ag NPs or $\mathrm{Ag}-\mathrm{Au}$ NPs, but this shrinkage is not enough to make $\mathrm{Ag}$ NPs or $\mathrm{Ag}-\mathrm{Au}$ NPs aggregate due to Ag NPs and Ag-Au NPs immobilized in the networks of the hydrogel. Generally, the aggregation of metal nanoparticles is irreversible. Here, the reversible variation of SPR with temperature change is possibly related to the reversible change of refractive index rather than the aggregation. The similar explanation for this phenomenon has been proposed in literature. ${ }^{59}$

\section{Catalytic activity of hybrid microgels}

The catalytic reduction of 4-NP by $\mathrm{NaBH}_{4}$ was monitored in situ by measuring the intensity of the absorbance peak of 4-NP at $400 \mathrm{~nm}$. In the absence of the catalyst, no absorption intensity change was observed after $30 \mathrm{~min}$ (ESI, Fig. S5†), indicating that the non-catalytic reduction of 4-NP hardly occurred.

When the reduction of 4-NP was performed in the presence of Ag-Au@P(NIPAM-co-MAPTMS) nanocatalysts with different Au contents (catalyst concentration $=0.15 \mathrm{~g} \mathrm{~L}^{-1}$, temperature $=$ 
$25{ }^{\circ} \mathrm{C}$ ), the above absorption intensity gradually decreased, and a new absorption band of 4-aminophenol appeared at $315 \mathrm{~nm}$ (Fig. 14) ${ }^{60}$ Simultaneously, the color of the reaction mixture changed from yellow to colorless. The time required for the complete reduction of 4-NP catalyzed by Ag@P(NIPAM-coMAPTMS) equaled $\sim 42$ min (Fig. 14a), decreasing to 24 and $17 \mathrm{~min}$ in cases of Ag-Au@P(NIPAM-co-MAPTMS)-2 and Ag$\mathrm{Au} @ \mathrm{P}(\mathrm{NIPAM}-\mathrm{co}$-MAPTMS)-3, respectively (Fig. 14b and c). Thus, the reaction rate was greatly improved by increasing the $\mathrm{Au}$ content of $\mathrm{Ag}-\mathrm{Au}$ bimetallic nanocomposites. ${ }^{61,62}$

The kinetics of the above catalytic reduction was investigated by plotting $\ln \left(A_{t} / A_{0}\right) v s$. reaction time (Fig. 14d) $\left(A_{t}\right.$ and $A_{0}$ denote absorbances at $400 \mathrm{~nm}$ at times $t$ and 0 , respectively). Taking into account the fact that $\mathrm{NaBH}_{4}$ was present in large excess with respect to 4 -NP, a pseudo-first-order model with respect to the latter was applied. The apparent rate constants $\left(k_{\text {app }}\right)$ determined from the linear plot of $\ln \left(A_{t} / A_{0}\right) v s$. reduction time at $25{ }^{\circ} \mathrm{C}$ equaled $0.062,0.123$, and $0.174 \mathrm{~min}^{-1}$ for Ag@P(NIPAM-co-MAPTMS), Ag-Au@P(NIPAM-co-MAPTMS)-2 and Ag-Au@P(NIPAM-co-MAPTMS)-3, respectively, confirming that the catalytic activity of Ag-Au@P(NIPAM-co-MAPTMS) bimetallic hybrid microgels significantly increased with increasing relative content of $\mathrm{Au}$. Moreover, the porous $\mathrm{Ag}-\mathrm{Au}$ bimetallic nanocomposites provided a large surface area for catalytic reaction, which significantly enhanced the reaction rate. ${ }^{63}$ Notably, the P(NIPAM-co-MAPTMS) shell not only improved the stability and dispersity of $\mathrm{Ag}-\mathrm{Au}$ bimetallic nanocomposites, but also provided a restricted reaction space for increasing the local concentration of the reaction substrate. Therefore, the temperature-dependent swelling and shrinkage of the above shell can be used to regulate catalytic activity.

To verify the temperature sensitivity of the reduction of 4-NP with $\mathrm{NaBH}_{4}$ in the presence of Ag-Au@P(NIPAM-coMAPTMS)-3 bimetallic hybrid microgels, their catalytic performances were evaluated at different temperatures. The
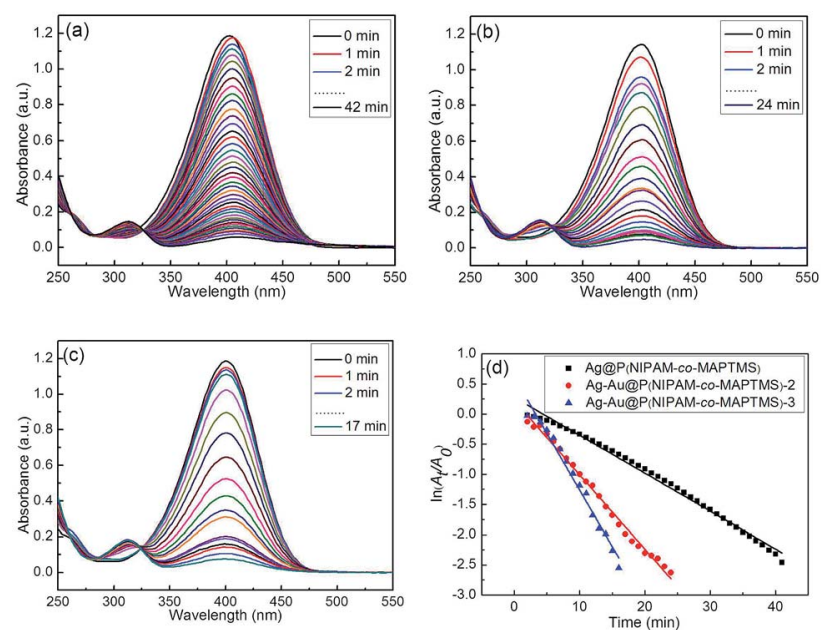

Fig. $14 U V$-Vis absorption spectra recorded during the reduction of 4-NP catalyzed by different nanocomposite catalysts $(a-c)$ and plots of $\ln \left(A_{t} / A_{0}\right)$ vs. time (d). (a) Ag@P(NIPAM-Co-MAPTMS), (b) AgAu@P(NIPAM-co-MAPTMS)-2, (c) Ag-Au@P(NIPAM-Co-MAPTMS)-3. corresponding UV-Vis absorption spectra for Ag-Au@P(NIPAM-co-MAPTMS)-3 (ESI, Fig. S6†) indicated that the reaction completion times equaled 17, 16, 19, 12, and $10 \mathrm{~min}$ at 25, 30, 32,35 , and $40{ }^{\circ} \mathrm{C}$, respectively, with the absence of a monotonous reaction rate increase implying that catalysis by $\mathrm{Ag}-$ Au@P(NIPAM-co-MAPTMS)-3 was inhibited at a certain temperature due to the thermosensitivity of P(NIPAM-coMAPTMS) microgels.

After a certain incubation time, $k_{\text {app }}$ values were determined at different reaction temperatures (Fig. 15a), equaling 0.174, $0.190,0.162,0.282$, and $0.320 \mathrm{~min}^{-1}$ for reduction catalyzed by Ag-Au@P(NIPAM-co-MAPTMS)-3 at 25, 30, 32, 35, and $40{ }^{\circ} \mathrm{C}$, respectively (Fig. 15b). Interestingly, the change of reaction rate with increasing temperature could not be well described by the Arrhenius equation, rather comprising three distinct stages. In the first stage, when the temperature was increased from 25 to $30{ }^{\circ} \mathrm{C}$, PNIPAM existed in an extended state, favoring the diffusion of reactants into the domains of $\mathrm{Ag}-\mathrm{Au}$ bimetallic nanocomposites. As a result, $k_{\text {app }}$ increased, and the catalytic reaction was accelerated. In the second stage, at temperatures (30 to $32{ }^{\circ} \mathrm{C}$ ) close to the LCST of PNIPAM, PNIPAM segments existed in a compact state, therefore inhibiting the contact of reactants with $\mathrm{Ag}-\mathrm{Au}$ and decreasing $k_{\text {app }}$. In the third stage (32 to $40{ }^{\circ} \mathrm{C}$ ), the increase of temperature overrode the above inhibitory effect, ${ }^{34}$ and the value of $k_{\text {app }}$ continued to increase. Thus, the above $k_{\text {app }}$-temperature correlation indicated that the chemical environment of $\mathrm{Ag}-\mathrm{Au}$ nanocomposites in $\mathrm{Ag}-$ Au@P(NIPAM-co-MAPTMS)-3 was significantly changed upon crossing the LCST threshold. Based on these results, we concluded that catalysis by Ag-Au@P(NIPAM-co-MAPTMS)-3 could be effectively controlled by changing the reaction temperature.

In order to further explore the effect of temperature on the $k_{\text {app }}$, the activation energy $\left(E_{\mathrm{a}}\right)$, Arrhenius factor $(A)$, enthalpy $(\Delta H)$ and entropy $(\Delta S)$ of activation for catalytic reduction of 4-NP before and after LCST was calculated according to Arrhenius and Eyring equation. ${ }^{64-66}$ As can be seen, the $E_{\text {a }}$ of before LCST and after LCST are $1.25 \times 10^{4} \mathrm{~J} \mathrm{~mol}^{-1}$ and $1.80 \times 10^{4} \mathrm{~J}$ $\mathrm{mol}^{-1}$, respectively (ESI Table S2 $\dagger$ ). The value of $A$ and $E_{\mathrm{a}}$ is greater at below LCST as compared to that at above LCST, which shows that catalytic reduction is more favourable at high temperatures as compared to low temperatures. The value of activation parameters at below LCST are $\Delta H=0.97 \times 10^{4}$ $\mathrm{J} \mathrm{mol}^{-1}$ and $\Delta S=-260.89 \mathrm{~J} \mathrm{~mol}^{-1} \mathrm{~K}^{-1}$, and that above LCST are
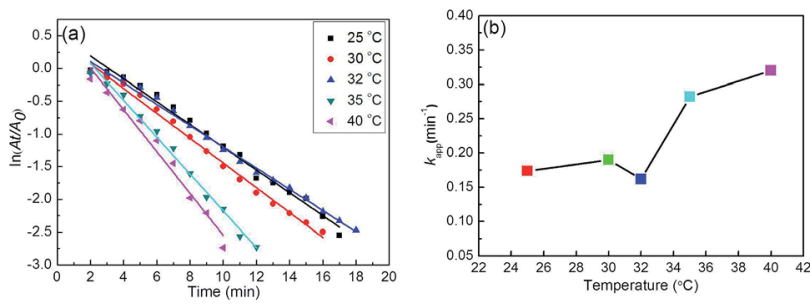

Fig. 15 Plots of $\ln \left(A_{t} / A_{0}\right)$ vs. time for the reduction of 4-NP catalyzed by Ag-Au(AP(NIPAM-CO-MAPTMS)-3 at different temperatures (a) and plot of $k_{\mathrm{app}}$ as a function of temperature (b). 
$\Delta H=1.39 \times 10^{4} \mathrm{~J} \mathrm{~mol}^{-1}, \Delta S=-244.60 \mathrm{~J} \mathrm{~mol}^{-1} \mathrm{~K}^{-1}$. It should be noted that the high value of $\Delta H$ and $\Delta S$ at above LCST suggests that reaction is more favourable at high temperatures as compared to that at low temperatures (below LCST). Above $35{ }^{\circ} \mathrm{C}$, the collapsed P(NIPAM-co-MAPTMS) organic-inorganic gel shell could be envisaged to require more energy for the reactant to close to nanocatalysts in the core part. It suggests that reaction is more favourable thermodynamically at high temperatures.

\section{Mechanism of catalytic activity regulation}

To explore the catalytic activity regulation mechanism of $\mathrm{Ag}-\mathrm{Au}$ bimetallic nanocomposites loaded with P(NIPAM-co-MAPTMS) organic-inorganic hybrid microgels, we compared the activities of Ag-Au@PNIPAM and Ag-Au@P(NIPAM-co-MAPTMS)-3 with identical contents of $\mathrm{Ag}-\mathrm{Au}$ nanocomposites at temperatures of 25,32 , and $40{ }^{\circ} \mathrm{C}$. As a result, the times required for the $\mathrm{Ag}-$ $\mathrm{Au} @$ PNIPAM catalyzed reduction of $4-\mathrm{NP}$ to proceed to completion were determined as 19,21 , and $13 \mathrm{~min}$ at 25, 32, and $40{ }^{\circ} \mathrm{C}$, respectively, with $k_{\text {app }}$ values determined as described above (ESI, Fig. S7 $\dagger$ ).

Fig. 16 shows the temperature dependence of $k_{\text {app }}$ for $\mathrm{Ag}$ Au@PNIPAM and Ag-Au@P(NIPAM-co-MAPTMS)-3, demonstrating that the latter catalyst exhibited higher activity than the former at identical temperatures, which showed that the introduction of the rigid MAPTMS into PNIPAM chains enhanced substrate mass transfer.

Upon a temperature increase from 25 to $32{ }^{\circ} \mathrm{C}$, the $k_{\text {app }}$ of $\mathrm{Ag}$ $\mathrm{Au} @ P N I P A M$ decreased from 0.150 to $0.117 \mathrm{~min}^{-1}$, while that of $\mathrm{Ag}-\mathrm{Au} @ \mathrm{P}(\mathrm{NIPAM}-\mathrm{co}$-MAPTMS)-3 decreased from 0.174 to $0.162 \mathrm{~min}^{-1}$. When the temperature was raised to $40{ }^{\circ} \mathrm{C}$, the reduction rate significantly increased in the case of $\mathrm{Ag}$ $\mathrm{Au} @ P(N I P A M-c o-M A P T M S)$. Compared to pure PNIPAM network chains, the swelling and shrinkage of P(NIPAM-coMAPTMS) organic-inorganic hybrid microgels was limited by the introduced rigid MPTMS segments. Close to its LCST, the PNIPAM shell layer encapsulating $\mathrm{Ag}-\mathrm{Au}$ bimetallic nanocomposites existed in a completely collapsed state, hindering contact between reactants and the metal catalyst. In $\mathrm{Ag}-$ $\mathrm{Au} @ P(N I P A M-c o$-MAPTMS), the thermosensitive PNIPAM chains were separated by rigid MAPTMS fragments, which inhibited the shrinkage of the former and allowed the facile diffusion of 4-NP and its contact with the $\mathrm{Ag}-\mathrm{Au}$ nanocatalyst.

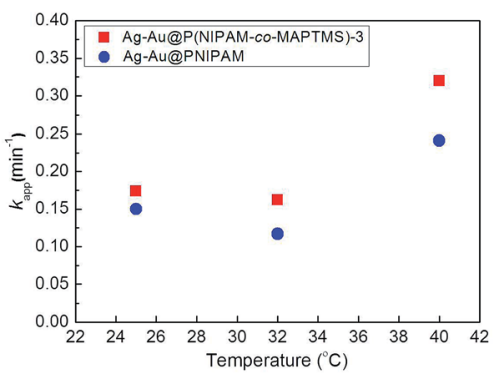

Fig. 16 Variation of $k_{\text {app }}$ with temperature for the reduction of 4-NP catalyzed by Ag-Au@P(NIPAM-co-MAPTMS)-3 and Ag-Au@PNIPAM.

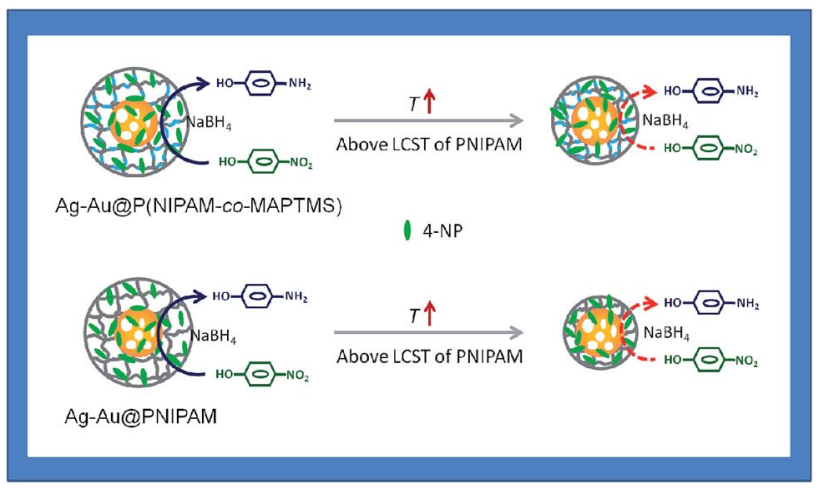

Fig. 17 The diagram of a possible catalytic activity regulation mechanism with Ag-Au@P(NIPAM-co-MAPTMS)-3 and Ag-Au@PNIPAM.

When the temperature was further increased to $40{ }^{\circ} \mathrm{C}, \mathrm{P}(\mathrm{NIPAM}-$ co-MAPTMS) hybrid microgels could easily maintain their highly porous structure, which favored substrate diffusion and increased its local concentration. Correspondingly, the enhanced permeability of the P(NIPAM-co-MAPTMS) organicinorganic layer shell could provide microdomains for the diffusion and mass transfer of the substrate to $\mathrm{Ag}-\mathrm{Au}$ bimetallic nanocomposites. Therefore, Ag-Au@P(NIPAM-co-MAPTMS) exhibited higher catalytic activity than Ag-Au@PNIPAM, with the above mechanism schematically illustrated in Fig. 17.

The comparison of apparent rate constant and intrinsic rate constant of our nanocatalytic systems to the literature recently reported on silver nanoparticles loaded hybrid microgels was presented in ESI (Table S3†). Comparing to other reports, our prepared $\mathrm{Ag}-\mathrm{Au}$ bimetallic nanocomposites stabilized with P(NIPAM-co-MAPTMS) organic-inorganic hybrid microgels exhibited higher catalytic activity for reduction of 4-NP below and above LCST. The reason is that three-dimensional structure of the P(NIPAM-co-MAPTMS) could provide microdomains for substrate diffusion and mass transfer due to the introduction of the rigid MAPTMS into PNIPAM network chains.

\section{Reusability of composite catalysts}

The stability of $\mathrm{Ag}-\mathrm{Au}$ bimetallic nanocatalysts was explored by determining the catalytic conversion of Ag-Au@P(NIPAM-coMAPTMS)-3 under typical conditions at $25{ }^{\circ} \mathrm{C}$ after re-cycling. The obtained results showed that although the above conversion decreased after four re-use cycles, it was still above $89 \%$ (ESI, Fig. S8 $\dagger$ ), with this slight reduction ascribed to the partial blockage of active sites. ${ }^{67}$ Thus, Ag-Au@P(NIPAM-co-MAPTMS) bimetallic hybrid microgel composites could serve as stable, efficient, and reusable catalysts.

\section{Conclusions}

In this study, we herein propose a novel route for the fabrication of porous $\mathrm{Ag}-\mathrm{Au}$ bimetallic nanocomposites stabilized by organic-inorganic hybrid microgels with temperature sensitivity, and these structural features are expected to enhance the catalytic reactivity of nanometal particles by intensification of 
the mass transfer. To verify the feasibility mentioned above, $\mathrm{Ag}-$ $\mathrm{Au}$ bimetallic nanocomposites stabilized with P(NIPAM-coMAPTMS) organic-inorganic hybrid microgels were prepared, and the reduction of 4-NP by $\mathrm{NaBH}_{4}$ catalyzed by the bimetallic hybrid microgels was performed. Based on the experiment result, the following conclusions can be drawn. (1) Introduction of rigid PMAPTMS segments into PNIPAM microgel could enhance the three-dimensional porous inflexibility of P(NIPAMco-MAPTMS), which is favor for the diffusion and mass transfer; (2) P(NIPAM-co-MAPTMS) could immobilize $\mathrm{Ag}-\mathrm{Au}$ bimetallic nanoparticles into network to inhibit the aggregation of metal nanoparticles; (3) both SPR and catalytic properties of $\mathrm{Ag}$ $\mathrm{Au} @ \mathrm{P}(\mathrm{NIPAM}-\mathrm{co}$-MAPTMS) are reversibly sensitive to external temperature due to presence of temperature sensitive PNIPAM chains. All conclusions aforementioned are significant to construction of hybrid microgels used in catalysis.

\section{Conflicts of interest}

There are no conflicts to declare.

\section{Acknowledgements}

This work was supported by the National Natural Science Foundation of China (21173141), Key Industry Project of Shaanxi Province of China (2011K08-14), National 111 Project of China (B14041), and Changjiang Scholars and Innovative Research Team in University of China (IRT1070).

\section{Notes and references}

1 R. L. S. Tan, X. H. Song, B. Chen, W. H. Chong, Y. Fang, H. Zhang, J. Wei and H. Y. Chen, Nanoscale, 2016, 8, 34473453.

2 Y. R. Wu, X. J. Sun, Y. Yang, J. M. Li, Y. Zhang and D. Qin, Acc. Chem. Res., 2017, 50, 1774-1784.

3 Z. Yin, Y. Wang, C. Q. Song, L. H. Zheng, N. Ma, X. Liu, S. W. Li, L. L. Lin, M. Z. Li, Y. Xu, W. Z. Li, G. Hu, Z. Y. Fang and D. Ma, J. Am. Chem. Soc., 2018, 140, 864-867.

4 H. Zhang, M. Jin and N. Y. Xia, Chem. Soc. Rev., 2012, 41, 8035-8049.

5 A. Q. Wang, X. Y. Liu, C. Y. Mou and T. Zhang, J. Catal., 2013, 308, 258-271.

6 K. D. Gilroy, A. Ruditskiy, H. C. Peng, D. Qin and Y. N. Xia, Chem. Rev., 2016, 116, 10414-10472.

7 J. W. Ahn, D. Wang, Y. Ding, J. W. Zhang and D. Qin, ACS Nano, 2018, 12, 298-307.

8 D. S. Wang and Y. D. Li, Adv. Mater., 2011, 23, 1044-1060.

9 W. G. Menezes, B. Neumann, V. Zielasek, K. Thiel and M. Bäumer, Phys. Chem. Chem. Phys., 2013, 14, 1577-1581.

10 A. Monga and B. Pal, New J. Chem., 2015, 39, 304-313.

11 M. B. Cortie and A. M. McDonagh, Chem. Rev., 2011, 111, 3713-3735.

12 M. K. Fan, F. J. Lai, H. L. Chou, W. T. Lu, B. J. Hwang and A. G. Brolo, Chem. Sci., 2013, 4, 509-515.

13 C. X. Li, C. Wang, Q. Y. Han, Y. N. Wu and H. R. Zheng, Plasmonics, 2017, 12, 1373-1379.
14 D. J. Wu and X. J. Liu, Appl. Phys. Lett., 2010, 97, 6190461911.

15 M. Ganguly, J. Jana, A. Palc and T. Pal, $R S C A d v .$, 2016, 6, 17683-17703.

16 P. N. Njoki, W. J. Wu, H. Zhao, L. Hutter, E. A. Schiff and M. M. Maye, J. Am. Chem. Soc., 2011, 133, 5224-5227.

17 A. Bansal, J. S. Sekhon and S. S. Verma, Plasmonics, 2014, 9, 143-150.

18 H. P. Liu, T. Z. Liu, L. Zhang, L. Han, C. B. Gao and Y. D. Yin, Adv. Funct. Mater., 2015, 25, 5435-5443.

19 Y. G. Sun and N. Y. Xia, Science, 2002, 298, 2176-2179.

20 Y. G. Sun and Y. N. Xia, J. Am. Chem. Soc., 2004, 126, 38923901.

21 L. M. Moreau, C. A. Schurman, S. Kewalramani, M. M. Shahjamali, C. A. Mirkin and M. J. Bedzyk, J. Am. Chem. Soc., 2017, 139, 12291-12298.

22 C. Z. Zhu, D. Du, A. Eychmüller and Y. H. Lin, Chem. Rev., 2015, 115, 8896-8943.

23 Y. C. Tsao, S. Rej, C. Y. Chiu and M. H. Huang, J. Am. Chem. Soc., 2014, 136, 396-404.

24 L. H. Yang, J. Lv, Y. M. Sui, W. Y. Fu, X. M. Zhou, J. W. Ma, S. Su, W. J. Zhang, P. Lv, D. Wu, Y. N. Mu and H. B. Yang, CrystEngComm, 2014, 16, 2298-2304.

25 O. L. Sánchez-Muñoz, J. Salgado, J. Martínez-Pastor and E. Jiménez-Villar, Nanosci. Nanotechnol., 2012, 2, 1-7.

26 M. Lin, Y. Q. Wang, X. Y. Sun, W. H. Wang and L. X. Chen, ACS Appl. Mater. Interfaces, 2015, 7, 7516-7525.

27 R. Contreras-Caceres, P. Alonso-Cristobal, D. MendezGonzalez, M. Laurenti, A. Maldonado-Valdivia, F. GarciaBlanco, E. L. Cabarcos, A. Fernandez-Barbero, J. M. LopezRomero and J. Rubio-Retama, Langmuir, 2014, 30, 1556015567.

28 X. Dong, X. B. Zou, X. Y. Liu, P. Lu, J. M. Yang, D. L. Lin, L. Zhang and L. S. Zha, Colloids Surf., A, 2014, 452, 46-50.

29 G. L. Li, C. A. Tai, K. G. Neoh, E. T. Kang and X. L. Yang, Polym. Chem., 2011, 2, 1368-1374.

30 S. Y. Chen, L. Y. Wang, X. Dong, X. Y. Liu, J. F. Zhou, J. M. Yang and L. S. Zha, RSC Adv., 2016, 6, 48479-48488.

31 C. F. Xiao, Q. S. Wu, A. P. Chang, Y. H. Peng, W. T. Xu and W. T. Wu, J. Mater. Chem. A, 2014, 2, 9514-9523.

32 H. G. Schild, Prog. Polym. Sci., 1992, 17, 163-249.

33 S. Carregal-Romero, N. J. Buurma, J. Pérez-Juste, L. M. LizMarzán and P. Hervés, Chem. Mater., 2010, 22, 3051-3059.

34 S. Wu, J. Dzubiella, J. Kaiser, M. Drechsler, X. H. Guo, M. Ballauff and Y. Lu, Angew. Chem., Int. Ed., 2012, 51, 2229-2233.

35 C. F. Xiao, S. M. Chen, L. Y. Zhang, S. Q. Zhou and W. T. Wu, Chem. Commun., 2012, 48, 11751-11753.

36 S. Y. Li, D. L. Lin, J. F. Zhou and L. S. Zha, J. Phys. Chem. C, 2016, 120, 4902-4908.

37 R. Begum, Z. H. Farooqi, E. Ahmed, K. Naseem, S. Ashraf, A. Sharif and R. Rehan, Appl. Organomet. Chem., 2017, 31, 1-8.

38 R. Begum, Z. H. Farooqi, Z. Butt, Q. S. Wu, W. T. Wu and A. Irfan, J. Environ. Sci., DOI: 10.1016/j.jes.2017.12.003.

39 Z. H. Farooqi, S. R. Khan and R. Begum, Mater. Sci. Technol., 2017, 33, 129-137. 
40 J. Zhao, L. F. Liu and Y. Zhang, Acta Phys.-Chim. Sin., 2015, 31, 1549-1558.

41 M. Y. Wang, R. Niu, M. Huang and Y. Zhang, Sci. China: Chem., 2015, 45, 76-89.

42 A. H. Li, J. Q. Liu, G. Z. Liu, J. Z. Zhang and S. Y. Feng, J. Polym. Sci., Part A: Polym. Chem., 2014, 52, 87-95.

43 P. Hervés, M. Pérez-Lorenzo, L. M. Liz-Marzán, J. Dzubiella, Y. Lu and M. Ballauff, Chem. Soc. Rev., 2012, 41, 5577-5587.

44 N. G. Bastus, F. Merkoci, J. Piella and V. Puntes, Chem. Mater., 2014, 26, 2836-2846.

45 X. Hong, D. S. Wang, R. Yu, H. Yan, Y. Sun, L. He, Z. Q. Niu, Q. Peng and Y. D. Li, Chem. Commun., 2011, 47, 5160-5162.

46 Y. Dong, Y. Ma, T. Y. Zhai, F. G. Shen, Y. Zeng, H. B. Fu and J. N. Yao, Macromol. Rapid Commun., 2007, 28, 2339-2345.

47 R. Contreras-Cáceres, A. Sánchez-Iglesias, M. Karg, I. Pastoriza-Santos, J. Pérez-Juste, J. Pacifico, T. Hellweg, A. Fernández-Barbero and L. M. Liz-Marzán, Adv. Mater., 2008, 20, 1666-1670.

48 K. Zhang, J. Ma, B. Zhang, S. Zhao, Y. P. Li, Y. X. Xu, W. X. Yu and J. Y. Wang, Mater. Lett., 2007, 61, 949-952.

49 F. Zhang and C. C. Wang, Colloid Polym. Sci., 2008, 286, 889895.

50 Y. Choi, S. Hong, L. C. Liu, S. K. Kim and S. Park, Langmuir, 2012, 28, 6670-6676.

51 T. K. Sau and C. J. Murphy, J. Am. Chem. Soc., 2004, 126, 8648-8649.

52 A. G. Garcia, P. P. Lopes, J. F. Gomes, C. Pires, E. B. Ferreira, R. G. M. Lucena, L. H. S. Gasparotto and G. Tremiliosi-Filho, New J. Chem., 2014, 38, 2865-2873.

53 J. Park, Y. Kim, C. Oh, S. Shin, Y. Kim, S. Oh and S. Kong, Mater. Res. Bull., 2005, 40, 271-280.
54 J. Li, C. Y. Liu and Y. Liu, J. Mater. Chem., 2012, 22, 84268430.

55 J. Sun, H. X. Wu and Y. D. Jin, Nanoscale, 2014, 6, 5449-5457. 56 C. S. O. Silva, R. P. Baptista, A. M. Santos, J. M. G. Martinho, J. M. S. Cabral and M. A. Taipa, Biotechnol. Lett., 2006, 28, 2019-2025.

57 N. Alissawi, V. Zaporojtchenko, T. Strunskus, I. Kocabas, V. S. K. Chakravadhanula, L. Kienle, D. Garbe-Schönberg and F. Faupe, Gold Bull., 2013, 46, 3-11.

58 W. S. Guo, R. Pleixats and A. Shafir, Chem.-Asian J., 2015, 10, 2437-2443.

59 Z. X. Qian, K. N. Guye, D. J. Masiello and D. S. Ginger, J. Phys. Chem. B, 2017, 121, 1092-1099.

60 E. Menumerov, R. A. Hughes and S. Neretina, Nano Lett., 2016, 16, 7791-7797.

61 X. W. Liu, D. S. Wang and Y. D. Li, Nano Today, 2012, 7, 448466.

62 H. L. Jiang, T. Akita, T. Ishida, M. Haruta and Q. Xu, J. Am. Chem. Soc., 2011, 133, 1304-1306.

63 K. Liu, Y. C. Bai, L. Zhang, Z. B. Yang, Q. K. Fan, H. Q. Zheng, Y. D. Yin and C. B. Gao, Nano Lett., 2016, 16, 3675-3681.

64 S. R. Khan, Z. H. Farooqi, W. Zaman, A. Ali, R. Begum, F. Kanwal and M. Siddiq, Mater. Chem. Phys., 2016, 171, 318-327.

65 R. Begum, R. Rehan, Z. H. Farooqi, Z. Butt and S. Ashraf, J. Nanopart. Res., 2016, 18, 231.

66 S. Demirci and N. Sahiner, Water, Air, Soil Pollut., 2015, 226, 64.

67 A. Davoodnia and A. Khojastehnezhad, J. Chin. Chem. Soc., 2012, 4, 57. 